\title{
Construção coletiva de um currículo por competência para a residência em Medicina de Família e Comunidade
}

\author{
Gustavo de Oliveira Figueiredo \\ Cirurgião-Dentista, Mestre em Educação em Ciências e Saúde, Doutor em \\ Psicologia da Comunicação. Professor Adjunto do Núcleo de Tecnologia \\ Educacional para Saúde da Universidade Federal do Rio de Janeiro \\ $\triangle$ gfigueiredo.ufrj@gmail.com \\ Valéria Ferreira Romano \\ Médica, Mestre e Doutora em Saúde Coletiva. Professora Adjunta da \\ Faculdade de Medicina da Universidade Federal do Rio de Janeiro
}

\section{Bruno Pereira Stelet}

Médico, Mestre em Saúde Coletiva. Professor da Faculdade de Medicina da Universidade Federal do Rio de Janeiro. Preceptor na Clínica da Família Victor Valla

\section{Jorge Esteves Teixeira Júnior}

Médico, Especialista em Medicina de Família e Comunidade. Professor da Faculdade de Medicina da Universidade Federal do Rio de Janeiro. Preceptor na Clínica da Família Victor Valla

\section{Resumo:}

Trata-se de um estudo de caso que apresenta o processo e o produto da construção de um currículo por competência, elaborado de forma compartilhada entre os Programas de Residência em Medicina de Família e Comunidade da Universidade Federal do Rio de Janeiro (UFRJ) e da Escola Nacional de Saúde Pública da Fundação Oswaldo Cruz (ENSP), desenvolvido em parceria com a Clínica da Família Victor Valla, no bairro de Manguinhos, Rio de Janeiro. A necessidade de reconfigurar o currículo da Residência em Medicina de Família e Comunidade da UFRJ e da ENSP foi uma resposta ao desejo de qualificação de um processo de ensino em serviço na Atenção Primária à Saúde, como uma demanda de solidificação do Sistema Único de Saúde (SUS). A metodologia utilizada foi a de construção coletiva por meio de ciclos de oficinas de currículo e ensino com um total de 64 horas de encontros presenciais, entre os meses de janeiro a março de 2014. Valorizou-se uma abordagem participativa na qual uma equipe multiprofissional de dezessete pessoas trabalhou coletivamente em todo o processo de construção do projeto políticopedagógico do currículo. Como resultado foi elaborado um currículo por competência, organizado em oito dimensões, que buscou interseções entre os campos da Medicina de Família e Comunidade, da Saúde Coletiva e da Educação em Saúde. Este artigo traduz, em prospecção, a expectativa de abertura ao diálogo, na esperança de que outros programas de residência também promovam a ressignificação de seus currículos e qualifiquem o debate sobre formação profissional para o SUS.

Palavras-chave: Educação em Saúde; Currículo; Residência Médica; Medicina de Família e Comunidade. 


\title{
The collective construction of a curriculum by competence for the residence in Family and Community Medicine
}

\begin{abstract}
:
This is a case study that presents the process and the product of the construction of a competencybased curriculum, elaborated in a shared way between the Residency Programs in Family and Community Medicine, Universidade Federal do Rio de Janeiro (UFRJ) and the Escola Nacional de Saúde Pública da Fundação Oswaldo Cruz (ENSP), developed in partnership with the Clínica da Família Victor Valla, in the neighborhood of Manguinhos, Rio de Janeiro. The need to reconfigure the curriculum of the Residency in Family and Community Medicine at UFRJ and ENSP was a response to the desire to qualify a teaching process in service in Primary Health Care, as a demand for solidification of the Unified Health System (SUS). The methodology used was that of collective construction through cycles of curriculum and teaching workshops shared during the registration of a total of 64 hours of face-toface meetings, between January and March 2014. A participatory approach was which a multiprofessional team of seventeen people worked collectively in the whole process of building the political-pedagogical project of the curriculum. As a result, a competency-based curriculum was organized in eight dimensions that sought intercession between the fields of Family and Community Medicine, Collective Health and Health Education. This article translates, in prospecting, the expectation of openness to dialogue, in the hope that other residency programs will also promote the resignification of their curriculum and qualify the debate on vocational training for SUS.

Keywords: Health Education, Curriculum; Medical Residence; Community and Family Medicine.

\section{Construcción colectiva de un curriculo para la competencia para la residencia en Medicina de Familia y Comunidad}

\section{Resumen:}

Este es un estudio de caso que muestra el proceso y el producto de la construcción de un currículo para la competencia, preparado de una manera compartida entre los programas de residencia en medicina familiar y comunitaria en la Universidad Federal de Río de Janeiro (UFRJ) y la Escuela Nacional de Salud Pública (ENSP), desarrollado en colaboración con la Clínica de la Familia Victor Valla, en el barrio de Manguinhos, Rio de Janeiro. La necesidad de volver a configurar el currículo de la Residencia en Medicina Familiar y Comunitaria de la UFRJ y ENSP fue una respuesta al deseo de la calificación de un proceso de enseñanza en el servicio de atención primaria de salud, como la demanda de solidificación para el Sistema Único de Salud (SUS). La metodología utilizada fue la construcción colectiva a través de ciclos de talleres curriculares y didácticas compartidos en todo el registro de un total de 64 horas de reuniones cara a cara, entre los meses de enero a marzo de 2014. Aprecia un enfoque participativo el que un equipo multidisciplinar de diecisiete personas trabajó en conjunto durante todo el proceso de construcción del proyecto político-pedagógico del currículo. Como resultado de ello se ha diseñado un currículo por competencia organizado en ocho dimensiones, que buscaban intercesiones entre los campos de la Medicina Familiar y Comunitaria, Salud Pública y Educación para la Salud. En este artículo se traduce prospección, la expectativa de apertura al diálogo, con la esperanza de que otros programas de residencia también promueven la reformulación de sus hojas de vida y calificar el debate sobre la formación profesional para el SUS.

Palabras clave: Educación en Salud; currículo; residencia medica; medicina de familia y comunidad.

\section{INTRODUÇÃO}

Este artigo apresenta o processo e o produto de construção coletiva do currículo por competência do Programa de Residência em Medicina de Família e Comunidade da Universidade Federal do Rio de Janeiro (UFRJ) e da Escola Nacional de Saúde Pública Sérgio Arouca da Fundação Oswaldo Cruz (ENSP) com a coordenação metodológica do 
Núcleo de Tecnologia Educacional para a Saúde (NUTES/UFRJ). Tais Programas de Residência funcionam de forma compartilhada entre a UFRJ e a ENSP, através de um convênio específico e da parceria estabelecida com o município do Rio de Janeiro; tendo seu campo de prática localizado na Clínica da Família Victor Valla (CFVV), no bairro de Manguinhos, Rio de Janeiro.

A inserção dos autores nos Programas de Residência em Medicina de Família e Comunidade da UFRJ e da ENSP permitiu compreender a importância de aprofundar a reflexão no campo da educação na medida em que repensar o planejamento pedagógico para atender às necessidades de formação de médicos qualificados para o Sistema Único de Saúde (SUS), tornou-se um objetivo. Assim, sentiu-se a necessidade de organizar um currículo estruturado, com uma definição de perfil por competência, com métodos educacionais adequados centrados no profissional egresso.

Se a construção de um currículo por competência em um programa de Residência Médica pode ser justificado pela necessidade de organização de um processo educacional sentido no cotidiano do trabalho pelas pessoas envolvidas na condução da Residência, é relevante salientar também que evidentes avanços na expansão de cobertura da Atenção Primária à Saúde (APS) no país tem justificado uma preocupação específica com a formação de Médicos de Família e Comunidade. O exemplo maior disto é a indução do Ministério da Saúde para a expansão de vagas nos Programas de Residência em Medicina de Família e Comunidade (PRMFC) (BRASIL, 2013).

No entanto e apesar da relevância no âmbito da produção científica e do reconhecimento da sua importância e competência em países desenvolvidos, a Medicina de Família e Comunidade (MFC) ainda não goza da mesma importância dentro do SUS. Segundo Giovanella (2008), essa dificuldade não é exclusiva do Brasil e está atrelada à incipiência dos sistemas de saúde comum aos países em desenvolvimento, onde a maior parte do cuidado em saúde é realizado em ambiente hospitalar, restando à APS papel minoritário e seletivo, onde terapia de reidratação oral, contracepção e vacinação constituem o cerne dos serviços oferecidos.

Os obstáculos para que o MFC ocupe o papel de protagonista na APS nestes países foi analisado por Ponka et al. (2015), que destacam três principais: a) Os MFC em diferentes países desempenham diversos papéis nos seus cenários de prática, resolvendo, 
em grande parte, problemas que poderiam ser solucionados por uma organização multidisciplinar do processo de trabalho; b) Pela falta de organização dos sistemas, não há uma regulamentação determinando qual a especialidade médica responsável pelo primeiro contato com os pacientes. Com isso, diversas especialidades médicas acabam competindo dentro da própria corporação médica, dificultando a coordenação do cuidado e fazendo com que a escolha dos profissionais por uma especialidade seja mais pautada pela lógica de mercado do que pela necessidade da população; c) Dentro de um ambiente com grande sobrecarga de trabalho e com fluxos dos serviços pouco organizados, a longitudinalidade e seus benefícios parecem não ser algo fácil de se atingir.

O fortalecimento da APS depende diretamente da formação de médicos especialistas em cuidados primários para atender às necessidades de saúde da população. Essa premissa reforça a ideia de que, para ser resolutivo na APS, o profissional precisa ser treinado e capacitado para tal. Estudantes e residentes precisam ter experiência na APS durante sua formação, como forma de se chegar à compreensão de que cuidado em saúde é resultado de um sistema estruturado e depende muito mais da coordenação dos diversos níveis de atenção do que de condutas clínicas isoladas (KIDD, 2013).

Habitualmente, o treinamento de médicos especialistas se dá pela imersão no cotidiano de serviços específicos, cuja metodologia de ensino é centrada na convivência e observação da prática de um médico supervisor mais experiente e especialista. Apesar das atividades práticas estruturarem o processo de aprendizado, espera-se que ocorra uma relação intrínseca entre prática e teoria.

Segundo dados do Ministério da Saúde (BRASIL, 2014), em fevereiro de 2013 existiam cerca de 35 mil equipes de Saúde da Família implantadas no Brasil e e em dezembro de 2016, cerca de 40.600 equipes implantadas (BRASIL, 2016). Em 2013, um estudo nacional divulgado pelo Conselho Federal de Medicina apontava que o número de especialistas em MFC era de aproximadamente 3,5 mil médicos, assim considerando a diretriz do Ministério da Saúde de que haja um médico por equipe implantada, estes dados comprovam a grande defasagem na proporção de especialistas por equipe de Saúde da Família. Até os anos 2015, muitas políticas indutoras para expansão da oferta de vagas direcionadas à residência em MFC foram implementadas, entretanto o esforço de uma melhora qualitativa nos programas de residência existentes ainda se constitui enorme desafio. Nesse sentido, iniciativas de reflexão sobre o processo educacional e de 
ressignificação do currículo parecem ser fundamentais para a transformação do cenário atual.

A título de exemplo, podemos citar o Programa Mais Médicos (BRASIL, 2013) que pretendia igualar o número de egressos dos cursos de graduação de medicina ao número de vagas de residência até o ano de 2018.

Em uma revisão sobre a produção bibliográfica nacional acerca dos temas "Residência Médica" e "Medicina de Família e Comunidade", constatou-se que a maioria dos artigos publicados aborda a temática das diretrizes gerais de formação para a Atenção Primária, abordando principalmente o perfil do egresso e os conteúdos que devem constar nos cursos de formação nos níveis de graduação e lato sensu. Identificou-se que somente alguns poucos artigos tratavam da questão do currículo por competências ou apontavam para uma Diretriz Curricular Nacional. Todavia, poucos artigos discutem o processo de construção de programas de ensino orientados por competência ou realizam análises críticas sobre a estrutura dos currículos que estão sendo desenvolvidos no âmbito dos programas de residência médica.

Damos destaque ao documento intitulado Currículo Baseado em Competências para Medicina de Familia e Comunidade, publicado em 2015 pela Sociedade Brasileira de Medicina de Família e Comunidade (SBMFC), que tem como objetivo servir de guia para os programas de residência na especialidade no país, assim como nos processos de certificação de especialistas realizados pela SBMFC. Vale notar que o processo de construção curricular compartilhado descrito no presente artigo, não contou com as contribuições do documento da SBMFC, uma vez que ocorreram em 2014, antes da publicação do documento da SBMFC. Por outro lado, a posteriori, foi possível notar muitos pontos de convergência dentre os objetivos pedagógicos de ambos.

Na revisão da literatura internacional, pode-se atentar para a numerosa produção de trabalhos sobre os currículos dos programas de residência em diversos países e também sobre a construção de parâmetros e diretrizes regionais, tal como acontece na comunidade europeia em que as discussões sobre a construção de currículos por competências são mais frequentes e parecem direcionar a construção dos PRMFC.

A Academia Europeia de Professores em Medicina de Família (EURACT), por exemplo, desenvolve diversas ações para promover o aprimoramento dos Programas em 
Residência Médica em Medicina de Família e Comunidade na Europa. Dentre elas, A Agenda Educacional do EURACT, publicado em 2006, define as competências nucleares para o MFC com o objetivo de ofertar uma estrutura pedagógica que sirva de base para o currículos e políticas de saúde (EURACT, 2006). As competências são apresentadas em seis eixos, sendo eles: Gestão de Cuidados Primários, Orientação Comunitária, Aptidões Específicas para a Resolução de Problemas, Abordagem Abrangente, Cuidados Centrados na Pessoa e Abordagem Holística. Este modelo é apresentado numa árvore de competências, em que estes eixos compõe as ramificações, folhas e caules, enquanto na raiz encontram-se atitude, contexto e ciência. Estes últimos representam os valores do MFC, a importância da compreensão do contexto da especialidade no sistema de saúde e da necessidade de produção científica contínua.

O uso deste documento por este grupo de autores se deu pela compreensão de que estes eixos de competências eram o produto de um longo trabalho de consenso na Europa, continente em que a prática de MFC está mais consolidada do que na América Latina. Reiteramos que até o momento do início das oficinas, em 2014, não havia sido publicado o Currículo Baseado em Competências para Medicina de Familia e Comunidade da SBMFC como supracitado. Ademais, foi uma opção destes autores utilizar a organização do currículo a partir de competências essenciais ou nucleares, entendendo que uma lista extensa de competências poderia tornar a construção e execução do currículo pouco viáveis. Foi necessária uma releitura do documento da Agenda Educacional do EURACT, a fim de adaptar a proposta de competências europeia `a realidade brasileira.

\section{CONTEXTO INSTITUCIONAL DO PROGRAMA COMPARTILHADO DE RESIDÊNCIA EM MEDICINA DE FAMÍLIA E COMUNIDADE DA UFRJ E DA ENSP}

A Escola Nacional de Saúde Pública (ENSP), por meio do Centro de Saúde Escola Germano Sinval Faria (CSEGSF), e a Universidade Federal do Rio de Janeiro (UFRJ), por meio da Faculdade de Medicina (FM-UFRJ) e do Hospital Universitário Clementino Fraga Filho, além do Núcleo de Tecnologia Educacional para Saúde (NUTES), participam ativamente, em parceria com a Secretaria Municipal de Saúde do Rio de Janeiro, da formulação de políticas públicas de saúde no país e historicamente desenvolvem 
programas inovadores para a formação de profissionais para atuação nas unidades básicas do Sistema Único de Saúde.

Em 1994, quando o Ministério de Saúde implantou o Programa de Saúde da Família a nível nacional como uma estratégia de reorientação do modelo de atenção à saúde, essas instituições já desenvolviam iniciativas experimentais e projetos pilotos nessa direção. No ano 2000, como uma atividade do programa de desenvolvimento local integrado de Manguinhos, a ENSP implantou duas equipes de Saúde da Família no CSEGSF que serviram de referência e de campo de prática e pesquisas para que, em 2004, em colaboração com a Secretaria Municipal de Saúde do Rio de Janeiro, pudesse se expandir para oito equipes. A partir de 2010, no bojo do projeto TEIAS (Território Integrado de Assistência à Saúde de Manguinhos), foi possível atingir a cobertura de $100 \%$ da população do território de Manguinhos com a consolidação de treze equipes de saúde da família.

Atualmente, seis dessas equipes funcionam fora das dependências físicas da Fiocruz e totalmente integradas ao território, trabalhando em uma unidade de saúde do município nomeada Clínica da Família Victor Valla. É exatamente nesta unidade de saúde onde se desenvolvem as atividades dos programas de residência em MFC que se constituem os casos do presente artigo.

Com base na experiência vivida no cotidiano dos programas de residência, algumas reflexões do grupo se transformaram em questões norteadoras para a reconstrução do currículo em questão, a saber: a) Estamos trabalhando com um currículo adequado às necessidades de formação de Médicos de Família e Comunidade?; b) Qual projeto políticopedagógico melhor nos compromete com as transformações esperadas para o perfil do egresso de nossa Residência?; c) Que competências profissionais precisam ser desenvolvidas no processo educativo e expressas no currículo com o intuito de exercitar uma medicina de família e comunidade que atenda às reais necessidades da população?; e d) Como desenvolver de forma coletiva e participativa um currículo por competências que oriente nossa ação educacional?

A partir dessas indagações, a preocupação central durante as oficinas pedagógicas foi a de evitar formatos curriculares autoritários que reforçassem modelos tecnoassistenciais centrados exclusivamente na doença; para tanto, foi importante problematizar o currículo que se desenvolvia e também a própria ação dos envolvidos 
enquanto educadores. Por outro lado, também foi preciso considerar que os residentesmédicos que ingressam em um PRMFC foram submetidos, na maioria das vezes, a uma graduação em medicina fragmentada e reducionista e aprenderam a desenvolver condutas quase sempre desvinculadas dos contextos psicossocial e ambiental em que vivem os pacientes atendidos.

Como afirma Romano (2006), de maneira geral, os residentes foram estimulados em sua graduação de medicina a consolidar em si próprios um certo distanciamento do cuidado que um dia desejaram prestar; chegando ao fim da graduação em medicina aprendendo a valorizar um diálogo apressado, ou ainda, aceitar a crença irreal de que a conversa se dá exclusivamente com a doença e não com o sujeito dela portador.

Diante desses desafios, foi-se reafirmando a necessidade de construir um programa de residência com objetivos específicos de conciliar a racionalidade biomédica à compreensão sócio histórica do território e da comunidade e à ressignificação dos sentidos sobre a medicina no âmbito da família. Nessa perspectiva, a elaboração da proposta curricular buscou intercessões entre os campos da Medicina de Família e Comunidade, da Saúde Coletiva e da Educação em Saúde, valorizando uma abordagem participativa na qual cerca de dezessete profissionais, entre eles residentes, médicos, professores universitários, preceptores, gestores dos serviços de saúde, pesquisadores do campo da educação e a coordenação dos cursos trabalharam coletivamente por cerca de 64 horas em todo o processo de construção do projeto político-pedagógico; materializado em um currículo organizado por competências profissionais agrupadas em oito dimensões.

\section{Currículo por competências}

A definição do que vem a ser um currículo por competências envolve diferentes abordagens e acepç̃oes. A noção de competência começou a ser utilizada na Europa nos anos de 1980 e sua origem decorre da organização do trabalho em face da crise do modelo taylorista e fordista diante dos processos de mundialização da economia, da flexibilização da produção e do acirramento da competição no mundo do trabalho. 
Para Ramos (2001), o modelo de competência concorre com o conceito de qualificação - conceito-chave para a sociologia do trabalho - e está relacionado ao potencial de oferta que uma determinada formação pode oferecer ao aluno. No entanto, de acordo com a autora, competência é a capacidade de resolver um problema em um determinado cenário e baseia-se nos resultados, devendo ser compreendida como a capacidade de articular e mobilizar conhecimentos, habilidades e atitudes postos em ação para resolver problemas e enfrentar situações de imprevisibilidade em uma dada situação concreta de trabalho e em um determinado contexto cultural.

Estruturar um programa de ensino baseado em competência pressupõe a análise do processo de trabalho dos atores na prática (no caso o MFC), a fim de determinar os conhecimentos, habilidades e atitudes existentes no cotidiano real para o exercício da função. Posteriormente, essas categorias passariam por um processo de normalização que consiste na especificação de uma capacidade de trabalho, incluindo, ao menos, a descrição do alcance dessa capacidade, os critérios para julgar a qualidade da capacidade, as evidências de que a capacidade foi alcançada e os conhecimentos e conteúdos necessários.

Adotamos esta noção de competência que está centrada no princípio de que padrões de excelência de determinada profissão representam uma construção social o que nos permite estabelecer diálogos entre saberes disciplinares legitimados e práticas concretas orientadas por projetos de transformação da realidade que, por sua vez, demandam a legitimação de novos saberes. No entanto, é fundamental que tal reconhecimento esteja embasado em uma concepção crítica de educação e a métodos de aprendizagem significativa.

\section{Aprendizagem significativa}

As teorias de aprendizagens construtivistas defendem que a aprendizagem seja um processo social e ativo e que os aprendizes constroem seus conhecimentos por meio de suas experiências pessoais em interação social com outros. Moreira (1999) considera que é possível se construir uma analogia entre o processo de internalização dos significados de instrumentos e signos, a partir da interação social, defendida por Vygotsky (1999), e o conceito de aprendizagem significativa de Ausubel (1968), em que o significado lógico dos 
materiais de aprendizagem devem se transformar em significado psicológico para que o aprendiz construa seu conhecimento de forma significativa.

Nessa perspectiva, os novos conhecimentos são melhor internalizados pelos sujeitos quando se relacionam com as estruturas cognitivas construídas ao longo de toda sua experiência de vida. Em outras palavras, todo conhecimento é passível de ressignificação e todo aprendiz já possui alguma ideia prévia sobre aquilo que aprende. Dialogar com os conhecimentos prévios adquiridos a partir de novos conhecimentos que são propostos possibilita a reconfiguração do pensamento e a construção de novos significados para os novos saberes.

Se considerarmos que educação e transformação coincidem, uma questão que emerge é o modo como ativar processos educativos capazes de permitir o exercício do pensamento, da capacidade criativa e da interação com o outro, eclodindo no aprendiz sentimentos de acolhimento, reconhecimento e pertencimento. Segundo Freire (1970) o educador deve respeitar a leitura de mundo do educando e tomá-la como ponto de partida para a compreensão do papel da curiosidade como um dos impulsos primários da produção do conhecimento.

A adoção dos princípios da aprendizagem significativa pressupõe a construção de um currículo constituído pelos princípios da reflexividade crítica e da autonomia, em que o aprendiz é agente ativo na construção de seu próprio conhecimento, refazendo significados e redefinindo sentidos de acordo com a representação da realidade, necessariamente construída com base em suas experiências prévias. É a partir dessa perspectiva que se propõe refletir sobre a residência médica como um meio educativo de formação do profissional inserido no processo de trabalho em saúde desenvolvido no cotidiano dos serviços de saúde.

\section{Residência médica e a formação profissional em serviço}

Os discursos dos órgãos oficiais com foco na Educação Médica afirmam que há uma necessidade de articulação entre a formação, o currículo, a pesquisa e o mundo do trabalho. Na Educação em Saúde, essa articulação consiste em diretriz formalmente valorizada - tanto nos fundamentos que constituem o Plano Nacional de Educação quanto 
no Conselho Nacional de Educação, ao propor as atuais Diretrizes Curriculares Nacionais do Curso de Graduação em Medicina (DCN) (BRASIL, 2013). Nas DCN, encontramos referência e entendimento partilhado sobre a necessidade de lidar com os desafios e demandas contemporâneas no processo saúde-doença nos "diferentes contextos socioeconômicos em que há de haver médicos para atender e assistir a população" (BRASIL, 2013).

Ao assumir que a existência de médicos para todos os seguimentos da sociedade é uma demanda urgente, a formação médica no Brasil precisa considerar as modificações nos perfis demográficos e epidemiológicos da população como escopo de ensino e de trabalho, além de incorporar tecnologias e práticas que respondam à importância das condições crônicas e do envelhecimento crescente e estejam em sintonia com a atualidade. Sendo assim, associando a formação médica por competência com o mundo do trabalho, compreendemos que práticas voltadas para e no SUS consideram a relevância de estar consoante às políticas e à garantia de direitos sociais, o que tem sido discutido e indicado há muito pelos estudiosos da Educação em Saúde.

No âmbito da APS, especificamente nos serviços organizados pela estratégia de Saúde da Família, encontramos capilaridade da equipe de saúde no território, facilitando a adesão e o vínculo do usuário, o que amplifica o sentido maior do trabalho, isto é, o Cuidado em Saúde. Portanto, ao garantir a integralidade e a longitudinalidade do cuidado na formação profissional do médico, a área de MFC vem se afirmando com uma centralidade estratégica já que a residência médica é considerada pelo próprio Ministério da Educação como elemento indissociável da graduação, como componente que agrega qualidade à formação profissional (BRASIL, 2013).

Figueiredo e Wimmer (2006) afirmam que adotar uma perspectiva de formação profissional em serviço e apostar no potencial formador do SUS é assumir também que o ensino e o serviço, sempre em parceria com a população, possam enfrentar as fragilidades que transitam entre a gestão, a infraestrutura e o processo de trabalho, aproveitando as potencialidades dessa articulação - encarada como um contínuo processo em construção. Dessa maneira, quais sentidos se deveriam aportar ao currículo?

\section{Os sentidos do currículo}

Para Paim e Almeida-Filho (2000), a revisão e o desenvolvimento curricular podem ser medidas necessárias para a atualização das instituições de ensino face à reorganização 
das práticas de saúde. No entanto, mudanças nos currículos costumam ser insuficientes para alterar o modo de produção e interação das pessoas nele envolvidas. Os autores afirmam que a difusão de ideologias modernizantes e inovações curriculares, ainda que relevantes para o componente cultural de um dado projeto ético-político de transformação do ensino, têm sido insuficientes para promover mudanças significativas e continuadas na formação profissional em saúde.

Nessa mesma perspectiva de análise, Silva (1999) adverte que o currículo está estritamente relacionado às estruturas econômicas e sociais mais amplas e que, portanto, não constitui um corpo neutro, inocente e desinteressado de conhecimentos. De maneira geral, a seleção de conteúdos e métodos educacionais que compõem o currículo é resultado de um processo que reflete interesses particulares das classes e grupos dominantes. Assim, ainda de acordo com o autor, no processo de construção curricular, deve-se perguntar: Quais interesses orientam a seleção de um conhecimento particular para a composição de um currículo? Quais as relações de poder envolvidas no processo de seleção que resultam na construção de um currículo em particular?

Em um esforço de se aproximar do que seria um "processo de desenvolvimento curricular crítico", Paim e Almeida-Filho (2000) afirmam que esse processo não decorreria de uma deposição acrítica de conteúdos ou disciplinas, mas de uma prática centrada em um tripé que contempla análises de situações concretas, de produção científica e de reflexão teóricoepistemológica. Em certa medida, para formar profissionais com senso crítico e comprometidos com a realidade social em que se insere, além do tripé proposto pelos autores, é necessária uma permanente produção de sentidos políticos da atuação no campo da saúde e um desvelamento de elementos do currículo oculto no cotidiano de formação.

De acordo com Figueiredo, Brito e Botazzo (2003), o currículo oculto é composto pelos hábitos, tradições e comportamentos cotidianos desenvolvidos nas instituições de ensino que reproduzem um modelo de Ciência na área de Saúde, que é tradicionalmente voltado para a construção de hipóteses, testagem de novos produtos, de novas técnicas, cedendo muito facilmente, tanto no meio acadêmico quanto na prática cotidiana, à pressão das grandes indústrias de fármacos e equipamentos médicos. Essa postura, historicamente, não vem auxiliando na solução dos problemas de saúde. Ao contrário, o modelo tem agravado as diferenças sociais e estimulado o crescimento do setor privado em detrimento do setor público de saúde. Tem, portanto, favorecido a venda e o consumo da saúde como um bem que vem sendo considerado uma mercadoria. 
Pires-Filho (1995) aborda a inadequação entre o currículo de formação dos profissionais de saúde e as expectativas da sociedade. Segundo o autor, a solução não está exatamente no perfil do profissional egresso, mas na própria concepção forjada a respeito do que seja currículo e do papel que se atribui à escola.

Numa certa perspectiva, a escola pode ser pensada como uma mera transmissora de saberes neutros, isto é, de valores incontestáveis, sem referência a seus contextos de produção ou de aplicação. Saberes cristalizados, anteriores à experiência, acima e à parte das relações sociais. Sob essa ótica, a escola não é vista como um campo de construção e reelaboração crítica do conhecimento, mas como local de consumo de saberes produzidos dentro e fora dela.

Compreender a educação como um processo capaz de desenvolver competências nos sujeitos para que construam autonomia de escolhas e de vida, exige um currículo em que o processo de ensino-aprendizagem proporcione ao educando reflexão crítica sobre sua prática profissional e sua responsabilidade frente às transformações sociais. Nesse caso, é preciso que o currículo - formal e oculto - estimule uma compreensão profunda da vida humana e da ligação que existe entre Educação, Política e o processo concreto e histórico de viver.

O desenvolvimento do currículo do PRMFC aqui abordado foi inspirado em Freire (1970, 1997), principalmente quando afirma: ensinar exige reconhecer que a educação é ideológica e muitas vezes os modelos pedagógicos tradicionais dominantes são reproduzidos e aplicados de forma acrítica em cursos e propostas curriculares que se autodenominam inovadoras, mas que, a despeito das inovações técnicas, reproduzem teorias, métodos e valores conservadores. Nesse sentido, é relevante assumir uma postura crítica diante do desenvolvimento de um currículo e reconhecer a importância das diferentes ideologias em conflito que, para Giroux (1986), mobilizam os sujeitos humanos e criam o terreno sobre o qual os homens se movimentam e adquirem consciência de sua posição na sociedade.

Isto posto, os autores deste estudo assumem uma posição atenta diante da construção do currículo em questão, entendendo que tal processo engendrava limitações políticopedagógicas que, de forma alguma, era preciso superar. Somente no reconhecimento de contradições, ambiguidades e paradoxos foi possível propor mudanças curriculares no PRMFC da UFRJ e da ENSP. 


\section{METOdOLOGIA DE DESENVOLVIMENTO DO CURRÍ́cUlO DO PROGRAMA DE RESIDÊNCIA EM MEDICINA DE FAMÍLIA E COMUNIDADE DA UFRJ E DA ENSP}

A metodologia utilizada para o desenvolvimento desta proposta baseou-se na ideia de constução compartilhada na educação em saúde desenvolvida por Brito e Siqueira (1993) e no método para o desenvolvimento de currículos baseado em competências profissionais (LIMA e RIBEIRO, 2003). Nesta proposta, coletivos interdisciplinares e intersetoriais se organizam para socializar experiências pessoais e desenvolver de forma conjunta as estratégias educativas. Essas experiências de construção coletiva na área da educação em saúde são potentes instrumentos que vêm sendo utilizados ao longo dos últimos anos. (PESSANHA e ROMEIRO, 2003; FERREIRA, COTTA e OLIVEIRA, 2009; CHIAVERINI, ACIOLI e FIGUEIREDO, 2009).

Em nosso processo específico, utilizamos a metodologia desenvolvida por Chiaverini, Acioli e Figueiredo (2009) quando propuseram um método coletivo e participativo para a construção do currículo e do material didático para o curso de especialização em saúde da família oferecido pelo Instituto de Medicina Social em parceria com a faculdade de enfermagem, ambos da Universidade do Estado do Rio de Janeiro com o apoio do Ministério da Saúde (ver quadro I). Os encontros para produção do currículo deste programa de formação compartilhado UFRJ-ENSP foram organizados adotando um processo de construção coletiva por meio de um ciclo de oficinas com os seguintes objetivos: i) elaborar, desenvolver e implementar uma proposta compartilhada de formação, integrando a expertise em Pesquisa e em Práticas de Saúde Pública da Fundação Oswaldo Cruz com a expertise de Ensino e formação em Medicina, especificamente em Medicina de Família e Comunidade, da Faculdade de Medicina da Universidade Federal do Rio de Janeiro; a expertise em metodologias educacionais e planejamento curricular do Laboratório de Currículo e Ensino do Núcleo de Tecnologia Educacional para Saúde também da Universidade Federal do Rio de Janeiro; ii) organizar e integrar eixos temáticos e estratégias metodológicas que articulassem teoria e prática, ensino e serviço, assistência clínica e promoção da saúde, cuidado médico e autonomia do usuário; iii) desenvolver, implementar e avaliar um projeto piloto de formação de médicos em Atenção Primária, analisando criticamente a experiência compartilhada de ensino e produzindo documentos e análises que representassem contribuições práticas para a construção de novos parâmetros para a formação médica no país; iv) construir métodos, estratégias e instrumentos de avaliação e monitoramento do programa de Residência considerando as necessidades dos residentes, dos professores, dos preceptores e dos usuários, além de considerar a possibilidade de avaliar constantemente o processo pedagógico do programa proposto. 
A elaboração do currículo valorizou uma metodologia participativa na qual dezessete pessoas, entre médicos, professores universitários que exercem função de preceptoria, gestores dos serviços de saúde, investigadores do campo da Saúde Pública, educadores e a equipe de coordenação da iniciativa debatessem conhecimentos, experiências e concepções ao longo de um ciclo de oito oficinas (gerais e específicas) que aconteceram entre janeiro e março de 2014, totalizando cerca de 64 horas de trabalho coletivo.

\section{Método do Ciclo de Oficinas para Planejamento Curricular}

As oficinas desenvolvidas foram denominadas de Oficinas Gerais e Específicas segundo os objetivos do encontro e o tipo de colaboração dos participantes envolvidos. Nas Oficinas Gerais, participaram de forma integrada todos os atores apresentados anteriormente, formando um coletivo de trabalho integrado construído por uma plenária de dezessete pessoas. Nas Oficinas Específicas, participou um grupo mais restrito formado pela coordenação da iniciativa e por mais três representantes eleitos na plenária geral. No quadro I, é realizada a descrição dos objetivos de cada um dos encontros do ciclo de oficinas.

Quadro 1 - Topologia de Rede Social

\begin{tabular}{|c|c|c|c|c|c|c|c|c|}
\hline & $\begin{array}{c}\text { OFICINA } \\
01\end{array}$ & $\begin{array}{l}\text { OFICINA } \\
02\end{array}$ & OFICINA & $\begin{array}{c}\text { OFICINA } \\
04\end{array}$ & $\begin{array}{c}\text { OFICINA } \\
05\end{array}$ & $\begin{array}{c}\text { OFICINA } \\
06\end{array}$ & $\begin{array}{c}\text { OFICINA } \\
07\end{array}$ & $\begin{array}{c}\text { OFICINA } \\
08\end{array}$ \\
\hline & GERAL & GERAL & GERAL & GERAL & ESPECÍFICA & GERAL & ESPECÍFICA & GERAL \\
\hline $\begin{array}{l}\text { O } \\
\text { B } \\
\text { J } \\
\text { E } \\
\text { T } \\
\text { I } \\
\text { V } \\
\text { O }\end{array}$ & $\begin{array}{l}\text { Identificar as } \\
\text { ações e } \\
\text { atividades da } \\
\text { prática em } \\
\text { medicina de } \\
\text { família e } \\
\text { comunidade }\end{array}$ & $\begin{array}{c}\text { Definir as } \\
\text { competências } \\
\text { gerais e } \\
\text { específicas } \\
\text { identificando os } \\
\text { principais } \\
\text { atributos } \\
\text { (Conhecimen- } \\
\text { tos, Habilidades } \\
\text { e Atitudes) }\end{array}$ & $\begin{array}{c}\text { Agrupar as } \\
\text { competências } \\
\text { em Dimensões } \\
\text { e desenvolver } \\
\text { os atributos } \\
\text { de cada uma } \\
\text { das } \\
\text { competências. }\end{array}$ & $\begin{array}{l}\text { Pactuação das } \\
\text { Competências } \\
\text { Transversais; e } \\
\text { Diagnóstico dos } \\
\text { principais } \\
\text { problemas da } \\
\text { prática em } \\
\text { medicina de } \\
\text { família e } \\
\text { comunidade. }\end{array}$ & $\begin{array}{l}\text { Elaboração de } \\
\text { um modelo de } \\
\text { roteiros de } \\
\text { atividades } \\
\text { educacionais e } \\
\text { organização } \\
\text { do currículo } \\
\text { do programa } \\
\text { de Residência. }\end{array}$ & $\begin{array}{c}\text { Primeira } \\
\text { Validação do } \\
\text { currículo } \\
\text { desenvolvido } \\
\text { e construção } \\
\text { do plano de } \\
\text { curso para os } \\
\text { dois anos do } \\
\text { programa de } \\
\text { residência. }\end{array}$ & $\begin{array}{c}\text { Análise e } \\
\text { Revisão dos } \\
\text { produtos } \\
\text { elaborados nas } \\
\text { oficinas } \\
\text { anteriores com } \\
\text { base na primeira } \\
\text { validação pelo } \\
\text { coletivo. }\end{array}$ & $\begin{array}{c}\text { Validação } \\
\text { final do } \\
\text { currículo e } \\
\text { organização } \\
\text { do processo } \\
\text { de trabalho de } \\
\text { professores, } \\
\text { preceptores e } \\
\text { residentes. }\end{array}$ \\
\hline
\end{tabular}

Fonte: Adaptado de CHIAVERINI; ACIOLI e FIGUEIREDO (2009).

Uma vez definidos os objetivos das oficinas e organizado o processo de trabalho dos encontros, foi iniciada a sistematização das mesmas através de rodas de conversas entre os participantes que identificaram, agruparam, classificaram e definiram as competências dos residentes em MFC, considerando para tal seus conhecimentos, habilidades e atitudes. Partindo do questionamento geral "que características esperamos de um Médico de Família e Comunidade?", construímos uma matriz de competência formatando nossas conclusões, mas entendendo a condição transitória da mesma, diante da inerente e constante necessidade de reavaliação. No quadro II, reproduzimos a matriz de competências desenvolvida. 
Quadro II - Matriz de Competências: Dimensões e Competências do Programa de Residência em Medicina de Família e Comunidade UFRJ/ENSP

PROGRAMA DE RESIDÊNCIA EM MEDICINA DE FAMÍLIA E COMUNIDADE UFRJ/ENSP

DIMENSÕES

\section{COMPETÊNCIAS}

\begin{tabular}{|c|c|}
\hline \multirow{7}{*}{$\begin{array}{c}\text { DIMENSÃO I: } \\
\text { GESTÃO DE CUIDADOS } \\
\text { PRIMÁRIOS EM SAÚDE }\end{array}$} & 1. Realizar o manejo clínico dos problemas prevalentes \\
\hline & 2. Coordenar o cuidado com a equipe interdisciplinar na rede de atenção à saúde \\
\hline & 3. Registrar, analisar e utilizar informações em saúde \\
\hline & 4. Organizar a consulta médica \\
\hline & 5. Desenvolver o seguimento longitudinal do cuidado \\
\hline & 6. Participar na organização do serviço de saúde \\
\hline & $\begin{array}{l}\text { 7. Assumir compromisso e responsabilizar-se pelo cuidado com as pessoas, as famílias e a } \\
\text { comunidade }\end{array}$ \\
\hline $\begin{array}{l}\text { DIMENSÃO II: ABORDAGEM } \\
\text { CENTRADA NA PESSOA }\end{array}$ & 1. Conhecer e aplicar diversas abordagens centradas na pessoa \\
\hline \multirow{6}{*}{$\begin{array}{c}\text { DIMENSÃO III: } \\
\text { RESOLUTIVIDADE DE } \\
\text { PROBLEMAS (INDIVIDUAL, } \\
\text { FAMILIAR E COLETIVO) }\end{array}$} & 1. Tomar decisões para a resolução de problemas \\
\hline & 2. Construir, implementar e monitorar Projetos Terapêuticos Singulares \\
\hline & 3. Trabalhar com a clínica em atenção primária \\
\hline & 4. Atender em situações de urgência e emergência \\
\hline & 5. Atuar considerando os princípios da prevenção quaternária \\
\hline & 6. Identificar recursos e articular a rede de atenção intersetorial \\
\hline \multirow{3}{*}{$\begin{array}{c}\text { DIMENSÃO IV: } \\
\text { ATENÇÃO INTEGRAL À SAÚDE }\end{array}$} & 1. Trabalhar com a concepção ampliada de saúde \\
\hline & 2. Trabalhar na perspectiva da promoção da saúde e desenvolvimento social \\
\hline & 3. Trabalhar na perspectiva de prevenção de agravos à saúde \\
\hline \multirow{6}{*}{$\begin{array}{c}\text { DIMENSÃO V: } \\
\text { ABORDAGEM COMUNITÁRIA E } \\
\text { PARTICIPAÇÃO POPULAR }\end{array}$} & 1. Conhecer a determinação social da saúde \\
\hline & 2. Compreender a representação social da saúde e da doença \\
\hline & 3. Reconhecer o território-processo identificando fragilidades e potencialidades \\
\hline & 4. Articular iniciativas de Educação Popular em Saúde \\
\hline & 5. Fomentar e apoiar a participação política engajando-se na comunidade \\
\hline & 6. Desenvolver visita domiciliar como estratégia de abordagem comunitária \\
\hline \multirow{3}{*}{$\begin{array}{l}\text { DIMENSÃO VI: ABORDAGEM } \\
\text { FAMILIAR E REDES DE APOIO } \\
\text { SOCIAL }\end{array}$} & 1. Reconhecer as possibilidades de arranjo e organização familiar \\
\hline & 2. Conhecer e utilizar ferramentas de abordagem familiar \\
\hline & 3. Conhecer e articular redes de apoio social \\
\hline \multirow{6}{*}{$\begin{array}{l}\text { DIMENSÃO VII: } \\
\text { TRABALHO EM EQUIPE }\end{array}$} & 1. Conhecer princípios de Gestão do Processo de Trabalho em equipe \\
\hline & 2. Desenvolver habilidades e atitudes para o trabalho em equipe \\
\hline & 3. Trabalhar na perspectiva de educação permanente \\
\hline & 4. Planejar e avaliar o trabalho em equipe \\
\hline & 5. Conhecer as atribuições das categorias profissionais \\
\hline & 6. Trabalhar com a lógica de apoio matricial \\
\hline \multirow{3}{*}{$\begin{array}{l}\text { DIMENSÃO VIII: } \\
\text { COMPETÊNCIAS } \\
\text { TRANSVERSAIS }\end{array}$} & 1. Trabalhar os aspectos contextuais na prática do Médico de Família Comunidade \\
\hline & 2. Desenvolver atitudes necessárias à prática do Médico de Família e Comunidade \\
\hline & 3. Desenvolver atividades de pesquisa e sistematização de saberes \\
\hline
\end{tabular}

Fonte: Elaboração própria dos autores (2014). 
Como pode ser observado no quadro II, foi proposta uma matriz de competências organizada em oito Dimensões: gestão de cuidados primários em saúde; abordagem centrada na pessoa; resolutividade de situações-problema (individual, familiar e coletiva); atenção integral à saúde; abordagem comunitária e participação popular; abordagem familiar e redes de apoio social; trabalho em equipe e as competências transversais: aspectos atitudinais, contextuais e produção científica.

Essa matriz de competências serviu de base para a organização de um currículo no qual um conjunto de competências se articulam entre si por meio de módulos educacionais com duração aproximada de três meses, evocando, assim, a perspectiva de integração de todos os conhecimentos, habilidades e atitudes necessários para a formação dos residentes em MFC, conforme indicado no quadro III a seguir. 


\begin{tabular}{|c|c|c|c|c|c|c|c|c|c|}
\hline \multicolumn{2}{|c|}{ QUADRO III: CURRÍCULO RESIDÊNCIA EM MEDICINA DE FAMÍLIA E COMUNIDADE UFRJ/ENSP } & $\begin{array}{l}\text { Módulo } \\
1\end{array}$ & $\begin{array}{l}\text { Módulo } \\
\quad 2\end{array}$ & $\begin{array}{l}\text { Módulo } \\
3\end{array}$ & $\begin{array}{l}\text { Módulo } \\
4\end{array}$ & $\begin{array}{l}\text { Módulo } \\
5\end{array}$ & $\begin{array}{l}\text { Módulo } \\
\quad 6\end{array}$ & $\begin{array}{l}\text { Módulo } \\
\quad 7\end{array}$ & $\begin{array}{l}\text { Módulo } \\
8\end{array}$ \\
\hline DIMENSÃO & COMPETÊNCIAS GERAIS & \multicolumn{4}{|c|}{ Primeiro Ano (R1) } & \multicolumn{4}{|c|}{ Segundo Ano (R2) } \\
\hline \multirow{7}{*}{$\begin{array}{c}\text { DIMENSÃO I: } \\
\text { GESTÃO DE CUIDADOS } \\
\text { PRIMÁRIOS EM SAÚDE }\end{array}$} & 1. Realizar o manejo clínico dos problemas prevalentes & $\mathbf{X}$ & $\mathbf{x}$ & $\mathbf{x}$ & $\mathbf{X}$ & $\mathbf{X}$ & $\mathbf{x}$ & $\mathbf{x}$ & $\mathbf{X}$ \\
\hline & $\begin{array}{l}\text { 2. Coordenar o cuidado com a equipe interdisciplinar na rede de atenção } \\
\text { à saúde }\end{array}$ & & & & & $\mathbf{x}$ & $\mathbf{x}$ & $\mathbf{x}$ & $\mathbf{X}$ \\
\hline & 3. Registrar, analisar e utilizar informações em saúde & $\mathbf{X}$ & & & & & & & \\
\hline & 4. Organizar a consulta médica & & & $\mathbf{x}$ & & & & & \\
\hline & 5. Desenvolver o seguimento longitudinal do cuidado & & & $\mathbf{X}$ & $\mathbf{X}$ & & & & \\
\hline & 6. Participar na organização do serviço de saúde & $\mathbf{x}$ & & & $\mathbf{X}$ & & & & \\
\hline & $\begin{array}{l}\text { 7. Assumir compromisso e responsabilizar-se pelo cuidado com as } \\
\text { pessoas, as famílias e a comunidade }\end{array}$ & & $\mathbf{X}$ & & & & $\mathbf{X}$ & & \\
\hline $\begin{array}{c}\text { DIMENSÃO II: } \\
\text { ABORDAGEM } \\
\text { CENTRADA NA PESSOA }\end{array}$ & 1. Conhecer e Aplicar diversas abordagens centradas na pessoa & $\mathbf{X}$ & $\mathbf{X}$ & $\mathbf{X}$ & $\mathbf{X}$ & $\mathbf{X}$ & $\mathbf{X}$ & $\mathbf{x}$ & $\mathbf{X}$ \\
\hline \multirow{6}{*}{$\begin{array}{c}\text { DIMENSÃO III: } \\
\text { RESOLUTIVIDADE DE } \\
\text { SITUAÇÕES- } \\
\text { PROBLEMA } \\
\text { (INDIVIDUAL, } \\
\text { FAMILIAR E COLETIVO) }\end{array}$} & 1. Tomar decisões para a resolução de situações-problema & & & & & $\mathbf{X}$ & & $\mathbf{X}$ & \\
\hline & 2. Construir, implementar e monitorar Projetos Terapêuticos Singulares & & & $\mathbf{X}$ & & & & & \\
\hline & 3. Trabalhar com a clínica em atenção primária & $\mathbf{X}$ & $\mathbf{X}$ & $\mathbf{x}$ & $\mathbf{X}$ & & & & \\
\hline & 4. Atender em situações de urgência e emergência & & & & $\mathbf{X}$ & $\mathbf{X}$ & & & \\
\hline & 5. Atuar considerando os princípios da prevenção quaternária & & & $\mathbf{x}$ & & & & & $\mathbf{X}$ \\
\hline & 6. Identificar recursos e articular a rede de atenção intersetorial & & & & & & $\mathbf{X}$ & $\mathbf{x}$ & \\
\hline \multirow{3}{*}{$\begin{array}{c}\text { DIMENSÃO IV: } \\
\text { ATENÇÃO INTEGRAL À } \\
\text { SAƯDE }\end{array}$} & 1. Trabalhar com a concepção ampliada de saúde & $\mathbf{x}$ & & & & $\mathbf{X}$ & & & \\
\hline & $\begin{array}{l}\text { 2. Trabalhar na perspectiva da promoção da saúde e desenvolvimento } \\
\text { social }\end{array}$ & & & $\mathbf{X}$ & & & & $\mathbf{X}$ & \\
\hline & 3. Trabalhar na perspectiva de prevenção de agravos à saúde & & $\mathbf{X}$ & $\mathbf{X}$ & & & & & \\
\hline
\end{tabular}




\begin{tabular}{|c|c|c|c|c|c|c|c|c|c|}
\hline \multicolumn{2}{|c|}{$\begin{array}{l}\text { QUADRO III: CURRICULO RESIDENCIA EM MEDICINA DE FAMÍLIA E COMUNIDADE UFRJ/ENSP } \\
\text { (continuação) }\end{array}$} & Módulo & $\begin{array}{l}\text { Módulo } \\
2\end{array}$ & $\begin{array}{l}\text { Módulo } \\
3\end{array}$ & $\begin{array}{c}\text { Módulo } \\
4\end{array}$ & $\begin{array}{l}\text { Módulo } \\
5\end{array}$ & $\begin{array}{c}\text { Módulo } \\
6\end{array}$ & $\begin{array}{l}\text { Módulo } \\
7\end{array}$ & $\begin{array}{c}\text { Módulo } \\
8\end{array}$ \\
\hline DIMENSAO & COMPETÊNCIAS GERAIS & \multicolumn{4}{|c|}{ Primeiro Ano (R1) } & \multicolumn{4}{|c|}{ Segundo Ano (R2) } \\
\hline \multirow{6}{*}{$\begin{array}{l}\text { DIMENSAO V: } \\
\text { ABORDAGEM } \\
\text { COMUNITÁRIA E } \\
\text { PARTICIPAÇÃO } \\
\text { POPULAR }\end{array}$} & 1. Conhecer a determinação social da saúde & & & & & $\mathbf{X}$ & $\mathbf{X}$ & $\mathbf{X}$ & $\mathbf{X}$ \\
\hline & 2. Compreender a representação social da saúde e da doença & & $\mathbf{X}$ & & & & $\mathbf{X}$ & & \\
\hline & $\begin{array}{l}\text { 3. Reconhecer o território-processo identificando fragilidades e } \\
\text { potencialidades }\end{array}$ & $\mathbf{X}$ & $\mathbf{X}$ & $\mathbf{X}$ & $\mathbf{X}$ & $\mathbf{X}$ & $\mathbf{X}$ & & \\
\hline & 4. Articular iniciativas de Educação Popular e Saúde & & & $\mathbf{X}$ & $\mathbf{X}$ & $\mathbf{X}$ & $\mathbf{X}$ & $\mathbf{X}$ & \\
\hline & 5. Fomentar e apoiar a participação política engajando-se na comunidade & & & & & $\mathbf{X}$ & $\mathbf{X}$ & $\mathbf{X}$ & $\mathbf{X}$ \\
\hline & $\begin{array}{l}\text { 6. Desenvolver visita domiciliar como estratégia de abordagem } \\
\text { comunitária }\end{array}$ & $\mathbf{X}$ & $\mathbf{X}$ & & & & & & \\
\hline \multirow{3}{*}{$\begin{array}{c}\text { DIMENSÃO VI: } \\
\text { ABORDAGEM } \\
\text { FAMILIAR E REDES DE } \\
\text { APOIO SOCIAL } \\
\end{array}$} & 1. Reconhecer as possibilidades de arranjo e organização familiar & & $\mathbf{X}$ & & & & & & \\
\hline & 2. Conhecer e utilizar ferramentas de abordagem familiar & & & $\mathbf{X}$ & & & & $\mathbf{X}$ & \\
\hline & 3. Conhecer e articular redes de apoio social & & $\mathbf{X}$ & $\mathbf{X}$ & & & & $\mathbf{X}$ & \\
\hline \multirow{6}{*}{$\begin{array}{l}\text { DIMENSÃO VII: } \\
\text { TRABALHO EM EQUIPE }\end{array}$} & 1. Conhecer princípios de Gestão do Processo de Trabalho em equipe & & & & $\mathbf{X}$ & & & & \\
\hline & 2. Desenvolver habilidades e atitudes para o trabalho em equipe & & $\mathbf{X}$ & $\mathbf{X}$ & & & $\mathbf{X}$ & $\mathbf{X}$ & \\
\hline & 3. Trabalhar na perspectiva de educação permanente & & & & & & & $\mathbf{X}$ & $\mathbf{X}$ \\
\hline & 4. Planejar e avaliar o trabalho em equipe & & & & $\mathbf{X}$ & $\mathbf{X}$ & & & \\
\hline & 5. Conhecer as atribuições das categorias profissionais & $\mathbf{X}$ & & & & & & & \\
\hline & 6. Trabalhar com a lógica de apoio matricial & & $\mathbf{X}$ & & & & & & \\
\hline \multirow{3}{*}{$\begin{array}{l}\text { DIMENSÃO VIII: } \\
\text { COMPETÊNCIAS } \\
\text { TRANSVERSAIS }\end{array}$} & $\begin{array}{l}\text { 1. Trabalhar os aspectos contextuais na prática do Médico de Família } \\
\text { Comunidade }\end{array}$ & $\mathbf{X}$ & $\mathbf{X}$ & $\mathbf{X}$ & $\mathbf{X}$ & $\mathbf{X}$ & $\mathbf{X}$ & $\mathbf{X}$ & $\mathbf{X}$ \\
\hline & $\begin{array}{l}\text { 2. Desenvolver atitudes necessárias à prática do Médico de Família e } \\
\text { Comunidade }\end{array}$ & $\mathbf{X}$ & $\mathbf{X}$ & $\mathbf{X}$ & $\mathbf{X}$ & $\mathbf{X}$ & $\mathbf{X}$ & $\mathbf{X}$ & $\mathbf{X}$ \\
\hline & 3. Desenvolver atividades de pesquisa e sistematização de saberes & $\mathbf{X}$ & $\mathbf{X}$ & $\mathbf{X}$ & $\mathbf{X}$ & $\mathbf{X}$ & $\mathbf{X}$ & $\mathbf{X}$ & $\mathbf{X}$ \\
\hline
\end{tabular}

Fonte: Elaboração própria dos autores (2014). 
Por meio do Quadro III, as Dimensões e Competências Gerais foram articuladas com problemas concretos advindos do processo de trabalho na Estratégia Saúde da Família e que possibilitaram o desenvolvimento de uma aprendizagem significativa. Dessa forma, para resolver os desafios educacionais, os residentes deverão utilizar como recursos - através das atividades propostas - concepções teórico-práticas de diversas Dimensões simultaneamente, com a intencionalidade de proporcionar um ambiente motivador e estimulante, com flexibilidade para facilitar o estudo contextualizado, autodirigido e orientado.

Vale ainda destacar que as competências gerais são como ideias-chave sobre o que se pretende realizar em um tipo específico de ação. Estas caracterizam-se por assumir um caráter não apenas técnico, mas essencialmente ético-político, e que favoreçam a ideia de autonomia, de abordagem sintética dos problemas e de flexibilidade. Ao assumir a perspectiva de que, quando colocado em prática, o currículo certamente será reconfigurado e readaptado pelos atores envolvidos diretamente no processo educativo. Isto pressupõe uma valorização do cotidiano pedagógico na interface entre os mundos do trabalho e do ensino para que o currículo não engesse a criatividade e a produção de novos saberes e práticas contextualizados.

No quadro III, pode-se observar que a Matriz é composta por uma Dimensão e uma Competência Geral, não aparecendo as Competências Específicas, também já trabalhadas e desenvolvidas pelo grupo, porém que não foram incluídas neste texto por limitações de espaço na paginação do artigo (será tema de um próximo artigo a ser publicado posteriormente). As Competências Específicas nada mais são do que uma decomposição sucessiva da Competência Geral e indicam uma função que contribui para o desenvolvimento de conhecimentos, habilidades e atitudes deve exercitar na sua prática.

As Dimensões, as Competências Gerais e as Competências Específicas traduzidas de forma detalhada as atividades educativas a serem propostas, auxiliam na identificação de estratégias pedagógicas que devem ser implementadas para o alcance dos objetivos de aprendizagem e tornam claros os processos e produtos educacionais do PRMFC, facilitando a construção de uma matriz de avaliação formativa que identifique o potencial de desenvolvimento que os residentes alcançam em cada etapa do percurso de formação.

\section{CONSIDERAÇÕES FINAIS}

o currículo desenvolvido - e aqui apresentado em suas características mais gerais assume a postura de promover o diálogo entre as Ciências da Saúde, as Ciências Sociais e as 
Ciências Humanas, integrando conhecimentos dos campos da Educação em Saúde, da Medicina de Família e Comunidade e da Saúde Coletiva com as necessidades de saúde da população. Tudo isso na direção de propor um modelo de formação crítico e reflexivo que se oponha ao modelo tecnocrático hegemônico. Para se chegar a essa proposta de currículo, foram fundamentais a construção coletiva e o esforço de diálogo entre profissionais de diferentes campos de conhecimento. Esse processo de comunicação foi marcado por desafios: muitas vezes o grupo precisou resinificar palavras e conceitos, a construir juntos novos sentidos e a compreender os processos de negociação e pactuação inerentes aos processos de construção coletiva.

O ciclo de oficinas de planejamento curricular mostrou-se importante ferramenta metodológica para a produção do currículo. Mesmo com tensões, disputas por sentidos, dificuldades de entendimento e problemas operacionais e concretos provenientes da complexa realidade em que operam os serviços de saúde no município do Rio de Janeiro (em sua maioria, geridos por organizações sociais), o encontro com sujeitos comprometidos com a construção de um processo de educação crítica e reflexiva foi um fator importante para manter a abertura ao diálogo e às negociações.

O currículo desenvolvido é considerado um produto inacabado e em permanente processo de reconstrução. Entretanto, este artigo teve como principal objetivo compartilhar a experiência e, quiçá, colaborar para o debate no campo da formação em saúde. Dessa maneira, abre-se mais um canal de comunicação entre todos aqueles que estão comprometidos com a criação de espaços potentes de formação de médicos para atuação na atenção primária no SUS.

\section{REFERÊNCIAS BIBLIOGRÁFICAS}

AUSUBEL, D.. Educational Psychology: A Cognitive View. New York: Holt, Rinehart \& Winston, 1968.

BRAID, L.M.; MACHADO, M.F.; ARANHA, A.C.. Estado de la arte de estudios sobre currículo de cursos de formación de profesionales en la área de la salud: un estudio a partir de artículos publicados entre 2005 y 2011. Interface - Comunic., Saude, Educ., v. 16, n. 42, p. 679-92, 2012.

BRASIL. Ministério da Educação.. Diretrizes Curriculares Nacionais do Curso de Graduação em Medicina. Brasília: Conselho Nacional de Educação, 2013.

BRASIL. Ministério da Educação.. Lei N. 13.005 de 25 de junho de 2014: Institui o Plano Nacional de Educação 2014-2024, 2014. Disponível em: <http://www.planalto.gov.br/CCIVIL 03/Ato20112014/2014/Lei/L13005.htm>. Acesso em: 28/10/14. 
BRASIL. Ministério Da Saúde.. Histórico de Cobertura do Programa de Saúde da Família. 2014. Disponível em: <http://dab.saude.gov.br/dab/historico cobertura sf/historico cobertura sf relatorio.php.>. Acesso em: 11/10/14.

BRASIL. Ministério da Saúde.. Lei N. 12.871, de 22 de outubro de 2013: Programa Mais Médicos. 2013. Disponível em: <http://www.planalto.gov.br/ccivil 03/ ato2011-2014/2013/Lei/L12871.htm>. Acesso em: $13 / 01 / 15$.

BRASIL. Ministério da Saúde. Secretaria de Assistência a Saúde. Departamento de Atenção Básica. Histórico de cobertura da Saúde da Família. Disponível em: http://dab.saude.gov.br/dab/historico_cobertura_sf/historico_cobertura_sf_relatorio.php Acesso em: 12 de dezembro de 2016.

BRITO, D.T.; SIQUEIRA, V.H.. Construção Coletiva e Utilização de Material Instrucional para o Ensino Médico: Uma Proposta de Integração Ensino Servico. Educacion Medica y Salud, v. 27, n. 1, p. 123-135, 1993.

CHIAVERINI, D.; ACIOLI, S.; FIGUEIREDO, GO.. Curso de Especialização em Saúde da Família: Livro Didático. Rio de Janeiro: CEPESC-UERJ, 2009.

CONSELHO FEDERAL DE MEDICINA.. Demografia Médica no Brasil: Cenários e indicadores de distribuição. CFM/CREMESP, v. 2, 2013.

EURACT.. Agenda Educacional do EURACT; 2006 Disponível em: http://www.apmgf.pt/ficheiros/AEE.pdf. Acesso realizado em 03 abr 2015

FERREIRA, M.L.; COTTA, R.M.; OLIVEIRA, M.S.. Construção coletiva de experiências inovadoras no processo ensino-aprendizagem na formação de profissionais da saúde. Rev. Bras. Educ. Med., v. 33, n. 2, p. 240-6, 2009.

FEUERWERKER, L.. Mudanças na educação médica e residência médica no Brasil. Interface - Comun Saúde, Educ. 1998;2(3):51-71.

FIGUEIREDO, G.O.; BRITO, D.T.; BOTAZZO, C.. Ideologia, fetiche e utopia na saúde: uma análise a partir da saúde bucal. Ciência e Saúde Coletiva, v. 8, n. 3, p. 753-763, 2003.

FIGUEIREDO, G.O.; WIMMER, G.F.. Ação Coletiva para a Qualidade de Vida - Autonomia, Transdisciplinaridade e Intersetorialidade. Ciência e Saúde Coletiva, v. 11, n. 1, p. 145-154, 2006.

FREIRE, P.. Pedagogia do Oprimido. Rio de Janeiro: Paz e terra, 1970.

FREIRE, P.. Pedagogia da autonomia: saberes necessários à prática educativa. Rio de Janeiro: Paz e terra, 1997.

GIOVANELLA, L.. Atenção Primária à Saúde seletiva ou abrangente?. Cad. Saúde Pública, Rio de Janeiro, v.24, supl.1, p.s21-s23, 2008.

GIROUX, H.. Teoria crítica e resistência em educação: para além das teorias de reprodução. Petrópolis, RJ: Vozes, 1986.

HAGER P.; GONSCI A.; ATHANASOU, J.. General issues about assessment of competence. Assessment \& Evaluation in Higher Education. v. 19, n. 1, p 3-15, 1994.

KIDD, M.. The Contribution of Family Medicine to Improving Health Systems: A Guidebook from the World Organization of Family Doctors. CRC Press; 2013. 
LIMA, V.; RIBEIRO, E.. Construção de programas de formação baseado em competências: Termo de Referência do projeto ENSP EM MOVIMENTO. Rio de Janeiro: ENSP/ FIOCRUZ, 2003.

MOREIRA, M.A.. Aprendizagem Significativa. 1a. Edição, Brasília, Ed. UnB, 1999

PAIM, J; ALMEIDA-FILHO, N.. A crise da saúde pública e a utopia da saúde coletiva. Salvador: Casa da Qualidade Editora, 2000.

PESSANHA, M.A.; ROMEIRO, E.. Ensp em Movimento: construindo um Programa de Formação orientado por Competência para o Sistema de Saúde/RELATÓRIO FINAL. Rio de Janeiro: OPAS/OMS/ENSP-FIOCRUZ, 2003.

PIAGET, J.. Abstração reflexionante. Porto Alegre: Artmed, 2005.

PINHEIRO, R.; MATTOS, R.A.. Os sentidos da integralidade na atenção e no cuidado à saúde. Rio de Janeiro: IMS/UERJ, 2001.

PIRES FILHO, F.M.. A construção interativa do saber e do fazer acadêmicos: o desafio da prática integral. Tese (doutorado). Niterói: Universidade Federal Fluminense, 1995.

PONKA, D.; ROULEAU, K.; ARYA, N.; REDWOOD-CAMPBELL L, WOOLLARD R, SIEDLECKI B, DUNIKOWSKI L.. Developing the evidentiary basis for family medicine in the global context: The Besrour Papers: a series on the state of family medicine in the world. Can Fam physician. v. 61, n.7 p. 596-600, 2015,

RAMOS, M.N.. A pedagogia das competências: autonomia ou adaptação?. São Paulo: Cortez, 2001.

ROMANO, V.F.. Certificação por competência para o Médico de Família: uma proposta em construção. Tese (Doutorado). Rio de Janeiro: Universidade do Estado do Rio de Janeiro, 2006.

SAMPAIO, P.G.. Residência em Medicina de Família e Comunidade: dois programas brasileiros. Dissertação (Mestrado). Rio de Janeiro: ENSP/FIOCRUZ, 2013.

SBMFC - Sociedade Brasileira de Medicina de Família e Comunidade. Currículo Baseado Em Competências Para Medicina de Família e Comunidade; 2015. Disponível em: http://www.sbmfc.org.br/media/Curriculo \%20Baseado\%20em\%20Competencias(1).pdf. Acesso realizado em $15 \mathrm{dez} 2016$.

SILVA, T.T.. Documentos de identidade: uma introdução às teorias do currículo. Belo Horizonte: Autêntica, 1999.

STREIT, D.S.; BARBOSA NETO, F; LAMPERT, J.B.; LEMOS, J.M.C.; BATISTA, N.A. (orgs.). Educação Médica: 10 Anos de Diretrizes Curriculares Nacionais. Rio de Janeiro: Associação Brasileira de Educação Médica, 2012.

TEIXEIRA, D.S.. Residência médica em medicina de família e comunidade no rio de janeiro: o lugar do cuidado na estrutura curricular. [Dissertação]. Rio de Janeiro: NUTES, Universidade Federal do Rio de Janeiro: 2009.

TESSER, C.D. (org.). Medicalização Social e atenção à saúde no SUS. São Paulo: Hucitec, 2010.

VILlELA, F.; ARCHANGELO, A.. Fundamentos da Escola Siginificativa. São Paulo: Edições Loyola, 2014.

VYGOTSKY, L.S.. A formação social da mente. São Paulo: Martins Fontes, 1999.

WONCA - EUROPE. Definition of Family Medicine. Disponível em: http://www.woncaeurope.org/_Acesso realizado em 03 abr 2015. 